\title{
Impacto de la clase invertida en la percepción, motivación y rendimiento académico de estudiantes universitarios
}

\author{
Cristina Mendaña-Cuervo* y Enrique López-González \\ Facultad de Ciencias Económicas y Empresariales, Universidad de León, Campus de Vegazana, s/n. León-España \\ (correo-e: cristina.mendana@unileon.es; enrique.lopez@unileon.es)
}

${ }^{*}$ Autor a quien debe ser dirigida la correspondencia.

Recibido Mar. 31, 2021; Aceptado Jun. 5, 2021; Versión final Jul. 7, 2021, Publicado Dic. 2021

\begin{abstract}
Resumen
Este trabajo intenta contrastar la utilidad de la metodología de clase invertida (flipped classroom), en aras a potenciar el aprendizaje activo y colaborativo propio de la nueva sociedad del siglo XXI. Se aportan y contrastan evidencias de su implementación en una asignatura del grado en administración y dirección de empresas de una universidad española. Los resultados muestran que: 1) la experiencia práctica ha mejorado las expectativas que los alumnos tenían a priori, lo cual tiene relación con la valoración de los alumnos y su posterior opinión sobre la metodología; 2) la comparación realizada entre los dos grupos (experimental y control) ha puesto de manifiesto que si bien el grupo experimental ha mejorado en la motivación y los resultados académicos, esto no ha sido estadísticamente significativo. Se concluye que la utilización de la metodología de aula invertida frente a la metodología tradicional ha supuesto mayor motivación de los estudiantes hacia la asignatura.
\end{abstract}

Palabras clave: aprendizaje activo; aprendizaje invertido; evaluación; expectativas; rendimiento académico; motivación

\section{The impact of flipped classroom on the perception, motivation, and academic results of university students}

\begin{abstract}
This study aims to assess the impact of a flipped classroom approach on active and collaborative learning, which are characteristic of the XXI century society. The methodology is applied during a business administration and management course at a university in Spain. The results show that: 1) practical activities improved a priori student expectations, which are linked with students' evaluations and opinions about the methodology; 2) comparing both groups (experimental and control) shows that the experimental group improves in both motivation and academic results, but this is not statistically significant. It is concluded that applying a flipped classroom methodology, when compared to traditional methods, increases student motivation for the course.
\end{abstract}

Keywords: activity learning; flipped learning; academic performance; motivation; evaluation; expectations 


\section{INTRODUCCIÓN}

En la actual sociedad de la atención, en entornos hiperconectados y procesos de digitalización avanzando exponencialmente, los estudiantes (millennials y nativos digitales) demandan un cambio en los procesos educativos tradicionales. De hecho, en las dos décadas de lo que va de siglo se ha producido un cambio en la percepción sobre el modo de aprender de la denominada "Generación NET" (Oblinger y Oblinger, 2005), que se desenvuelve de manera cómoda, autónoma y natural con la información multimedia, que consume datos simultáneamente de múltiples fuentes y que espera respuestas inmediatas "sin distancias". Asimismo, cabe recordar que ya en la esencia del Espacio Europeo de Educación Superior (EEES) se planteaba que la docencia debería centrarse en el estudiante más que en el profesor: es el propio estudiante quien, tras la activación de un conjunto de competencias propiciadas por el diseño de la práctica educativa que efectúa el profesor-facilitador, debe tomar las riendas de su proceso de aprendizaje.

Adicionalmente, con la implantación de los estudios de Grado, se ha ampliado el abanico de opciones para el proceso de evaluación por parte de los estudiantes (evaluación continua, evaluación única final, compensación, programa Erasmus...). En este contexto, los autores han observado un incremento en el número de estudiantes que no realizan un seguimiento del proceso de evaluación continua, lo que se traduce en un abandono de la materia o en dificultades para superar dicho proceso de evaluación, con el consiguiente incremento en el porcentaje de calificaciones "No superados". Surge así la necesidad de innovar con alguna metodología que provoque una mayor implicación o motivación de los estudiantes, buscando un compromiso por su parte que suponga un mayor seguimiento de la asignatura y, probablemente, una disminución en la tasa de abandono y una mejora en los resultados académicos. En este contexto, se plantea la oportunidad de emplear metodologías activas y colaborativas que potencien este tipo de aprendizaje, entre las que se encuentra la denominada "Clase Invertida" o en el anglicismo generalizado de Flipped Classroom (FC) (Bergmann y Sams, 2012), cuya utilización está emanando en diferentes niveles educativos, siendo común ver esta metodología activa en las Universidades, tanto españolas como mundiales.

La clase invertida es un modelo pedagógico que supone girar la dinámica activa del proceso de aprendizaje, anticipando el trabajo de los alumnos al propio desarrollo docente en el aula de clase. Se traslada así el protagonismo a los estudiantes, buscando su implicación y participación activa, enfocando el tiempo en clase a las cuestiones prácticas u operativas suscitadas por los aspectos conceptuales previamente sugeridos por el profesor. De esta forma, se voltea el clásico proceso formativo, transmutándose ahora en la lección en casa y los deberes en clase, al objeto de optimizar pedagógicamente el tiempo de clase.

A los efectos de este trabajo, la situación actual en este tópico se aborda atendiendo a los tres aspectos siguientes. En primer término, en relación a la aplicabilidad de esta metodología, no son pocos los trabajos que realizan una revisión de la misma en el contexto universitario en general (Cheng et al., 2019; Lundin et al., 2018; Rivero-Guerra, 2018) así como concretamente en el sistema universitario español (Flores et al., 2016; Galindo-Domínguez y Bezanilla, 2019). En segundo lugar, el interés por esta metodología queda también puesto de manifiesto en trabajos que analizan la literatura sobre la misma como signo claro de la incursión del FC en el quehacer del profesorado, tanto desde el punto de vista de las publicaciones como sobre los tópicos abordados en las mismas (Little, 2015; Uzunboylu y Karagozlu, 2015). Y, en tercer lugar, enfocándonos en un área particular de la educación superior, concretamente en el ámbito de la Contabilidad, igualmente esta metodología activa participa de interés académico. La literatura al respecto tanto desde la perspectiva de su aplicación práctica como sobre los resultados que se obtienen con la misma, pueden encontrarse en trabajos en el ámbito anglosajón (Brown et al., 2016; Jamaludin et al., 2016) y también en el sistema universitario español (Lento, 2016; Ros Clemente et al., 2018; Prieto et al., 2021).

Sin embargo, en este trabajo se parte de la idea de que cualquier cambio metodológico debería conllevar un estudio en profundidad de los resultados que propicia, al asumir que el hecho de modificar una metodología tradicional por una metodología activa debería implicar necesariamente evaluar cómo ha afectado dicho cambio a los principales destinatarios, no solo desde el punto de vista de su predisposición sino también en los resultados emocionales o motivacionales que ha favorecido y en el resultado final, que mayoritariamente viene determinado por los resultados académicos obtenidos, si bien pudieran plantearse otras opciones para evaluar el rendimiento académico (Gutiérrez-Monsalve et al., 2021). De hecho, varios autores subrayan la necesidad de una mayor investigación sobre las percepciones de los estudiantes sobre el uso de FC (Roach, 2014; Uzunboylu y Karagozlu, 2015), ya que las expectativas positivas hacia una tarea influyen tanto en el proceso de aprendizaje, fundamentalmente en el compromiso, como en los resultados obtenidos.

En consecuencia, en el presente trabajo, en primer lugar, se aportan evidencias de los resultados de la implementación práctica de la metodología FC en el contexto de educación superior, concretamente en la asignatura "Contabilidad de Costes" del Grado en Administración y Dirección de Empresas de la Universidad de León, evaluando aspectos motivacionales y resultados académicos obtenidos en estudiantes sometidos a 
esta metodología frente a un grupo control con metodología tradicional. En segundo lugar, a través de un cuestionario validado, se pretende contribuir con el contraste estadístico de sus efectos en el cumplimiento de las expectativas que dicha metodología propicia en los alumnos.

Este trabajo se estructura entonces como sigue: Tras esta introducción, se realiza una breve descripción de la experiencia en la implementación y puesta en práctica de la metodología activa objeto de estudio. A partir de ahí, se establecen los objetivos que permiten proponer las preguntas de investigación, se expone la metodología utilizada y se describe y desarrolla el análisis realizado con los datos obtenidos. En base a lo anterior, se detallan los principales resultados obtenidos para, por último, establecer las conclusiones e implicaciones que pueden extraerse de dichos resultados.

\section{DISEÑO E IMPLEMENTACIÓN}

tigación educativa actual parece demostrar que, si los estudiantes tienen la oportunidad de revisar los conceptos teóricos clave antes de la clase, la sesión presencial se puede utilizar de manera más efectiva para el aprendizaje activo mediante el análisis y sobre todo la aplicación práctica de dichos conceptos (Bergmann y Sams, 2012). A este respecto, la puesta en práctica del FC implica, por tanto, un cambio en el diseño metodológico, variando la dirección en la que el docente plantea el proceso (Figura 1), ya que se trata de combinar la instrucción directa con el trabajo previo de los alumnos, de forma que se puedan apoyar todas las fases del ciclo de aprendizaje en consonancia con la Taxonomía de Bloom, si bien volteando la pirámide de los procesos cognitivos que configuran dicha taxonomía.

Progreso de aprendizaje en esta dirección

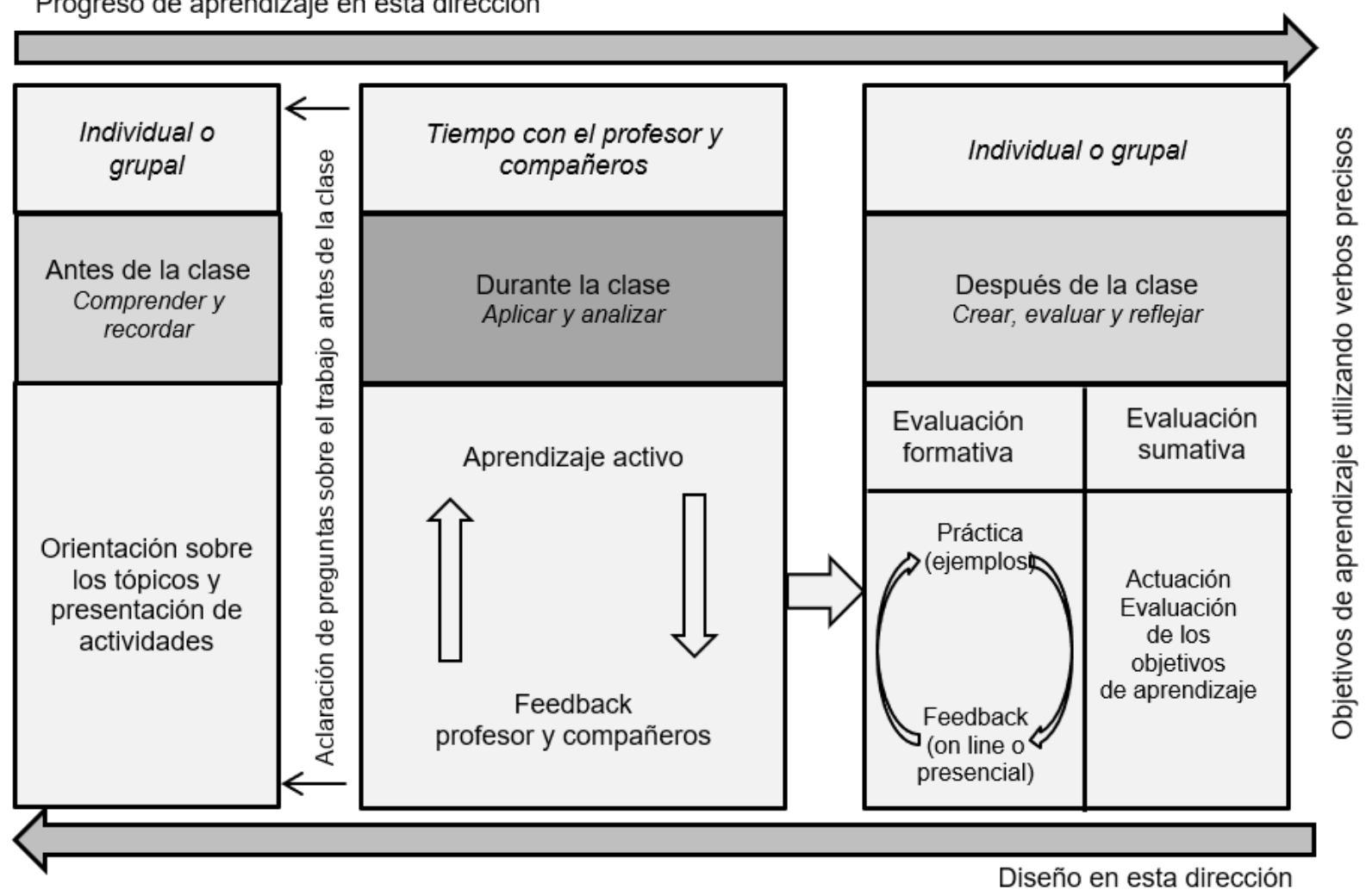

Fig. 1: Marco de diseño de FC alineado con la taxonomía modificada de Bloom, traducido y adaptado de Karanicolas, Snelling y Kemp (2018)

Por tanto, el proceso de implantación de la metodología FC precisa un nuevo diseño metodológico, no solo por los distintos materiales que han de formar parte del corpus de trabajo de los alumnos, sino también por los efectos que se espera provocar con los mismos. En este trabajo, como se indicó, la experiencia a que hace referencia que se enmarca en la asignatura "Contabilidad de Costes", se llevó a cabo de forma gradual en varios cursos académicos, en consonancia con la sugerencia de Steed (2012) respecto a que dicho proceso de puesta en práctica sea incremental, que se comience con una sola lección y se vayan comprobando resultados. Así, en el curso 2016/2017 la experiencia se circunscribió al primer tema del programa de la asignatura, lo que suponía un primer acercamiento tanto a la nueva metodología como a los contenidos fundamentales de la materia. Al objeto de evaluarla, se precisó mantener a los alumnos separados en dos grupos al azar (grupo experimental -GE- y grupo control -GC-), analizando los resultados a medida de la disposición de datos, para contrastar si resultaba factible su aplicación en toda la asignatura. A partir del 
curso 2017/2018 se amplió para las dos primeras partes del programa docente de la asignatura, continuando en el curso siguiente. En el curso 2019-2020 debido a la incidencia de la Covid-19 y la necesaria impartición a distancia, si bien se mantuvo esta metodología, no parece adecuado la comparación de los resultados obtenidos. Asimismo, fue necesario diseñar e implementar nuevos recursos docentes, en consonancia con la Figura 1 anterior, para abordar los distintos momentos de tiempo, tal como se muestra en la Figura 2.
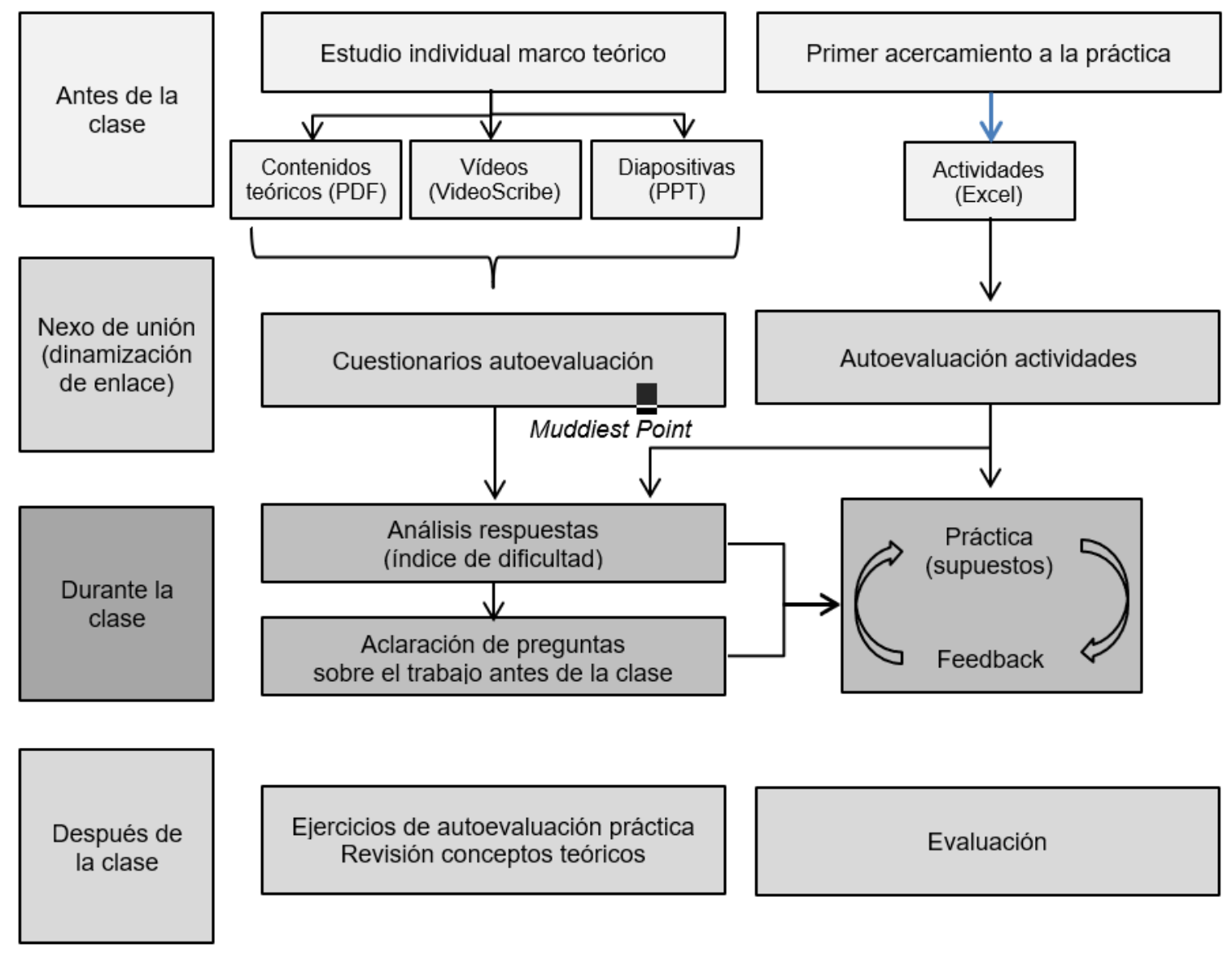

Ejercicios de autoevaluación práctica Revisión conceptos teóricos

Evaluación

Fig. 2: Diseño de FC "Contabilidad de Costes"

En relación a la elaboración de estos materiales, como cabía esperar y aunque la representación de las partes constitutivas de la Figura 2 pudieran parecer similares, conviene resaltar que el mayor esfuerzo e importancia relativa radicó en el diseño de la fase "Antes de la clase", esto es, los materiales puestos a disposición de los alumnos. Si bien es cierto que son varias las posibilidades, se ha optado por la implementación de vídeos, ya que su uso resulta común en sus prácticas cotidianas. También son varios los trabajos que analizan la satisfacción de los estudiantes en relación al uso de vídeos en su proceso de aprendizaje (Nagy, 2018), a las preferencias de los estudiantes sobre la tipología de los vídeos que preferentemente se utilizan en este proceso (Sohrabi y Iraj, 2016; Tse et al., 2019) así como comparativas con otro tipo de materiales a utilizar como contenido previo a la clase (Jensen et al., 2018). En aras a propiciar la predisposición de los alumnos hacia su visionado, los vídeos han sido elaborados por los propios profesores, utilizando el software VideoScribe, que permite apoyar con imágenes, textos, gráficos, etc., la explicación oral del propio profesor.

Lógicamente, los recursos docentes no se restringen a los vídeos, sino que son apoyados con otros recursos ya existentes, como pueden ser los contenidos teóricos de la asignatura o las presentaciones en PowerPoint que habitualmente se utilizan en clases presenciales, así como con otros recursos elaborados al respecto para un primer acercamiento a cuestiones prácticas. El material utilizado previo a la sesión presencial tiene su nexo de unión con el trabajo a realizar en el aula-clase, ya que para que este enfoque tenga éxito se ha de tener confianza en que los alumnos aprendieron la información y las habilidades necesarias para las actividades de aprendizaje activo antes de llegar al aula (Jensen et al., 2018, p. 523). En el estudio, se optó por ejercicios de autoevaluación de los alumnos sobre los tópicos trabajados de forma autónoma (a través de cuestionarios de autoevaluación de los conceptos teóricos) y ejercicios básicos de autoevaluación práctica, en consonancia con estudios que platean que una tarea breve o un cuestionario previo a la clase puede garantizar una preparación adecuada (Kim et al., 2014, p. 40), servir de incentivo (McNally et al., 2017, p. 281) y permitir identificar áreas comunes de dificultad o grupos de experiencia dentro de la clase (Abeysekera y Dawson, 2015, p. 10). En concreto, en el caso de los aspectos teóricos, se elaboró un banco de preguntas 
asociado a cada uno de los vídeos, de forma que cada alumno accede a un cuestionario con preguntas similares pero diferentes, con el fin de disponer de respuestas concretas y diferentes sobre los tópicos abordados en cada vídeo.

La utilización de cuestionarios permite obtener una información a disposición del profesor en la sesión presencial, a través del índice de dificultad que queda puesto de manifiesto en los mismos y que sirve de indicador de aquellos aspectos que han resultado más ambiguos y que pudieran necesitar una mayor dedicación o detalle en el aula de clase. Con este mismo fin, en todos los cuestionarios se ha utilizado una pregunta para determinar el aspecto más confuso (muddiest point) de cada uno de ellos (Mosteller, 1989), y cuya verificación sirve también de guía sobre los aspectos a incidir en la sesión presencial. Se pretende así que las actividades/discusiones en clase animen a alumnos y profesores a participar en un proceso continuo de recepción retroalimentación (Limniou et al., 2018), superando las dificultades achacadas a la metodología de FC en relación a que los estudiantes no comprendan o no participen en las actividades previas a la clase, a través de una visión general al inicio de la clase por parte del profesor sobre los aspectos más confusos o sobre los que se detecten mayores dificultades promoviendo un proceso de pensamiento de orden superior (Zainuddin y Halili, 2016).

En relación al proceso de autoevaluación práctica, se han diseñado ejercicios asociados también a cuestionarios y/o lecciones que permiten al estudiante corroborar sus conocimientos a medida que va calculando las soluciones a los supuestos planteados. Por otra parte, la modificación en la metodología implica que el tiempo de aula se deba aplicar de forma diferente, por lo que también ha sido preciso elaborar actividades de trabajo eminentemente prácticas, con las que apoyar y contrastar el aprendizaje que los alumnos han llevado a cabo en su espacio individual. Finalmente, tras el tiempo del aula, se han propuesto ejercicios más completos de autoevaluación eminentemente prácticos que permitan consolidar conceptos y que puedan servir de guía en aras a la evaluación de contenidos. Este enfoque centrado en datos permite desarrollar iniciativas de profundización particular o personalizada del proceso docente, al disponer de información sobre la participación activa de los alumnos en el visionado de vídeos y, de esta forma, poder recopilar datos para su análisis sobre los cuestionarios de autoevaluación y del resto de actividades, dotando al profesor de información valiosa sobre el desempeño docente.

\section{METODOLOGÍA}

El objetivo principal de este trabajo radica en evaluar las consecuencias derivadas de la implementación de la metodología activa FC en la motivación y resultados académicos obtenidos, así como conocer si las expectativas que genera en los estudiantes esta propuesta, mejoran o no con la experiencia. Este planteamiento da lugar a las siguientes preguntas de investigación: $\mathrm{Pl}_{1}$ : ¿La metodología FC aumenta la motivación de los estudiantes?, $\mathrm{Pl}_{2}$ : ¿La metodología FC aumenta el rendimiento académico de los estudiantes? y $\mathrm{Pl}_{3}$ : ¿La percepción de los estudiantes sobre la metodología FC mejora con la experiencia?. Adicionalmente, en este último caso, el diseño de las pruebas se llevará a cabo de forma que permita realizar a mayores un análisis sobre la percepción de los alumnos en relación a dos cuestiones: aspectos actitudinales y motivacionales (frente a metodologías tradicionales) y desarrollo de competencias genéricas que se asume va a potenciar la nueva metodología de forma prioritaria. De esta forma, se pueden plantear las dos siguientes sub-preguntas de investigación: $\mathrm{Pl}_{3-1}$ : ¿La percepción de los estudiantes con la metodología $\mathrm{FC}$ mejora en relación a aspectos actitudinales y motivacionales? y $\mathrm{Pl}_{3-2}$ ¿La percepción de los estudiantes con la metodología FC mejora en relación a las competencias adquiridas?

\section{Participantes}

El contexto en el que se ha desarrollado la experiencia atiende a las siguientes circunstancias: la población son los alumnos de la Facultad de Ciencias Económicas y Empresariales, Universidad de León (España), siendo la muestra de conveniencia aquellos que cursan la asignatura "Contabilidad de Costes", obligatoria de 3er curso del Grado en Administración y Dirección de Empresas (carga crediticia 6 ECTs) cuya edad se encuentra comprendida entre los 20 y 22 años y que para los periodos de estudio (cursos académicos 20162017, 2017-2018 y 2018-2019) los estudiantes han sido un total de 121 (67 varones y 54 mujeres), 100 (49 varones y 51 mujeres) y 87 (40 varones y 47 mujeres), respectivamente.

\section{Recolección de datos}

La recogida de información y recolección de datos se ha realizado a diferentes niveles y en distintos momentos de tiempo, teniendo en cuenta la dualidad de objetivos propuesta. Así, para evaluar las implicaciones en la motivación y el rendimiento académico de los estudiantes se ha considerado necesario poder establecer una comparación entre alumnos del mismo curso y año, pero en las dos situaciones posibles: metodología tradicional (GC) y metodología FC (GE). En este sentido, como método de evaluación se plantea dentro de los diseños experimentales un "diseño postest con grupo control" que se representa en la Figura 3. Para ello, 
Ios alumnos fueron distribuidos al azar en dos grupos, de forma que en el grupo experimental (GE) la docencia se desarrolló basándose en la metodología de clase invertida (FC), mientras que en el grupo de control (GC) se continuó con la metodología tradicional basada principalmente en clases magistrales. Una vez finalizada la programación docente, en ambos grupos se realizó una misma prueba de evaluación continua sobre los contenidos, obteniendo como observaciones los resultados de la prueba de evaluación única $(\mathrm{E})$.

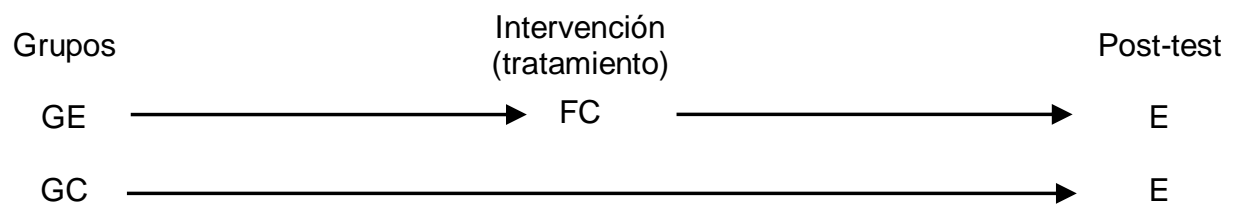

Fig. 3: Diseño de la experiencia

Con la finalidad de comprobar la motivación del alumnado, se compararon los porcentajes de alumnos que se presentaron a la prueba de evaluación continua en cada uno de los grupos (GE y GC), asumiendo que los alumnos a los que se les aplica la nueva metodología se han sentido más motivados en la asignatura. En relación al rendimiento académico, se trata de comprobar si las calificaciones de la prueba de evaluación realizada han supuesto una diferencia significativa entre el GE y GC. Por otro lado, al objeto de poder conocer la valoración de la metodología FC por parte de los estudiantes en primer término se diseñó un cuestionario ad hoc, denominado "Percepción de la metodología aula invertida" (

Tabla 1), sometiendo a análisis las propiedades psicométricas del mismo: en primer lugar, la validez de contenido por expertos y, en segundo lugar, la fiabilidad a través del método de consistencia interna alfa de Cronbach, cuyo detalle se recoge en Poy-Castro et al. (2017). Como se ha comentado, la validez de contenido y la fiabilidad fueron las propiedades analizadas para comprobar que el instrumento mide aquello que pretende medir y que la estabilidad temporal de las puntuaciones obtenidas es adecuada.

Tabla 1: Cuestionario "Percepción de la metodología aula Invertida"

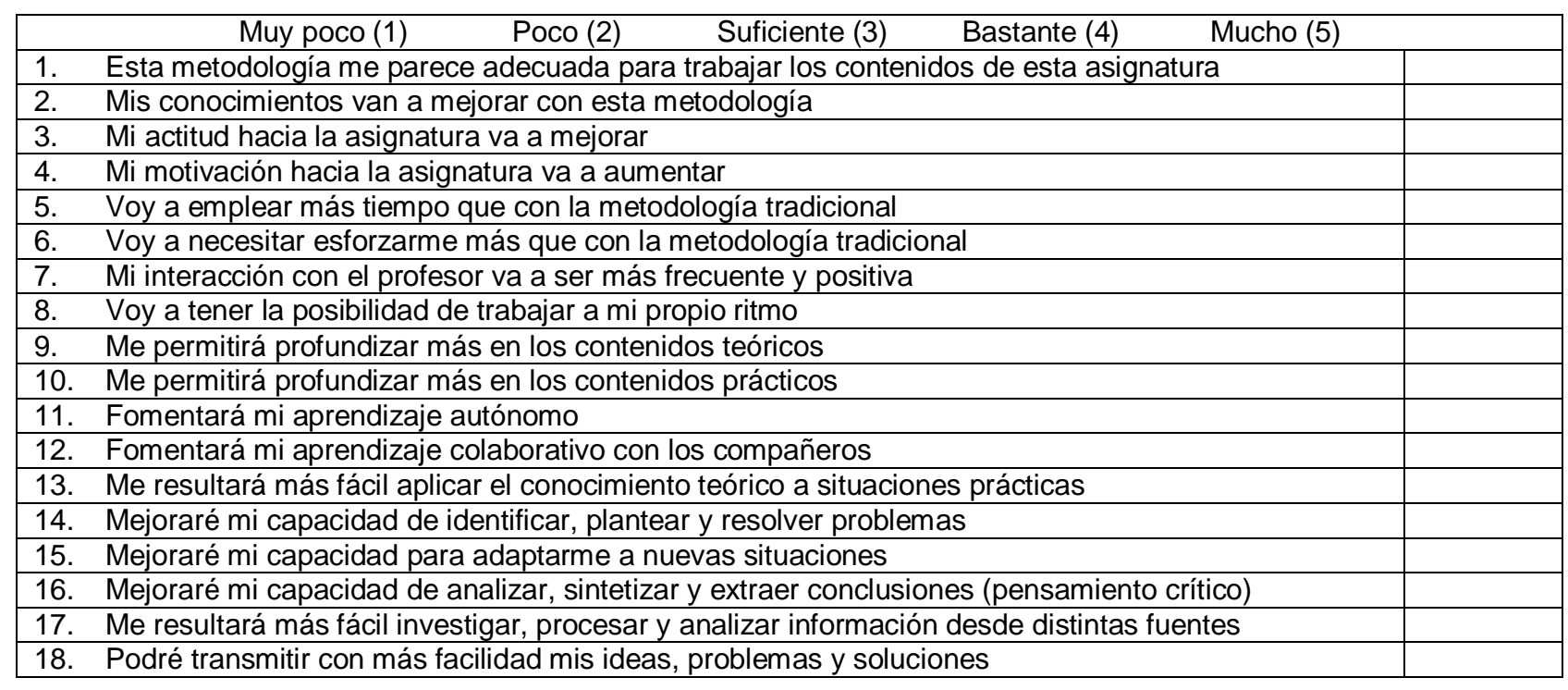

Dicho cuestionario se puso a disposición de los estudiantes sometidos a la metodología (GE) en la web institucional soportada por Moodle, cumplimentándolo antes de la experiencia, lo que permitió recabar la opinión de los alumnos a priori, cuando únicamente se les expuso el funcionamiento, objetivos y propósito de la metodología FC. Posteriormente, dicho cuestionario fue sometido a la consideración de los mismos alumnos una vez realizada la experiencia, permitiendo así obtener información sobre su percepción del funcionamiento real de esta metodología. Las cuestiones recogidas en el cuestionario final son idénticas al inicial, únicamente se efectuaron dos modificaciones. En primer lugar, se procedió a la redacción de los ítems en tiempo pasado, dado que la metodología ya había sido experimentada por dichos estudiantes. En segundo lugar, se añadió un ítem ("Me gustaría mantener esta metodología frente a la metodología tradicional en el resto del curso") para que los estudiantes pudiesen manifestar su opinión acerca de la posibilidad de implementar la metodología en el resto de la asignatura.

El hecho de contar con las opiniones iniciales de los estudiantes (expectativas) y su valoración final (opinión tras la experiencia) permite comparar esa información a fin de conocer si la implementación práctica del aula invertida (FC) puede considerarse como una experiencia positiva y extensible al resto de la materia o si, por 
el contrario, las expectativas generadas no se han visto corroboradas con la experiencia práctica. A este respecto, y como se ha comentado anteriormente, el diseño del cuestionario se llevó a cabo agrupando los ítems en dos categorías (Tabla 2) en relación a dos cuestiones: aspectos actitudinales y motivacionales (frente a metodologías tradicionales) y desarrollo de competencias genéricas, por lo que el estudio y análisis de resultados se ha realizado para cada uno de los dos grupos de ítems.

Tabla 2: Grupos de ítems a considerar en la evaluación de la percepción del alumnado a propósito del FC

\begin{tabular}{|l|c|}
\hline \multicolumn{1}{|c|}{ Aspectos a considerar } & Ítems \\
\hline G1. Cuestiones actitudinales y motivacionales & $1-3-4-5-6-7$ \\
\hline G2. Competencias adquiridas & $2-8-9-10-11-12-13-14-15-16-17-18$ \\
\hline
\end{tabular}

\section{Análisis de datos}

Para el análisis de los datos se han utilizado técnicas estadísticas clásicas para comprobar los supuestos de normalidad y homocedasticidad de las diferentes muestras y poder establecer las pruebas a utilizar para el caso de comparación de medias (o medianas) de los datos obtenidos en cada grupo propuesto. En el cuestionario los datos han sido obtenidos de la web institucional (Moodle) mientras que el resto de la información necesaria ha sido obtenida de las pruebas de evaluación realizadas, habiendo sido procesados en SPSS y/o Microsoft Excel.

\section{RESULTADOS Y DISCUSIÓN}

La exposición de resultados y la discusión se lleva a cabo tratando de dar respuesta a las preguntas de investigación planteadas de forma separada en cada uno de los subapartados siguientes. En relación a la discusión de los resultados obtenidos, dado que se trata de un estudio de caso concreto, se ha optado por utilizar referencias a trabajos recopilatorios de los resultados que se discuten.

\section{Incidencia en la motivación de los estudiantes $\left(P I_{1}\right)$}

Como se comentó con anterioridad, el análisis de datos en este caso se ha planteado en base al porcentaje de alumnos de cada grupo (GE/GC) presentados a la prueba de evaluación continua, si bien es cierto que la motivación del alumnado puede (y quizás debe) medirse a través de otras muchas variables. Sin embargo, desde el punto de vista de cuantitativo se puede considerar que esa información puede ser válida para establecer la comparación que se pretende. En la Tabla 3 se recogen los datos obtenidos para los tres cursos académicos analizados, separados por grupos de alumnos (GE/GC) y para las pruebas de evaluación práctica y teórica, de forma que, de acuerdo con lo anterior, para cada curso y prueba se trata de valorar: $\mathrm{H}_{0}$ : La proporción de alumnos presentados es la misma en el GE y en el GC

En las pruebas de evaluación práctica, con los datos obtenidos, el resultado del contraste de hipótesis de igualdad de proporciones en dos muestras independientes da como valor del estadístico $Z=2,18437$ y $p$ valor 0,01447 para el curso 2016/2017, estadístico $Z=1,96877$ y $p$-valor 0,02449 para el curso 2017/2018 y estadístico $Z=3,75906$ y p-valor 0,00009 en el curso 2018/2019. Por tanto, los resultados obtenidos, para un nivel de significación de $5 \%$, permiten afirmar que el número de alumnos presentados no es el mismo en ambos grupos, asumiendo que la motivación de los alumnos medida en estos términos mejora significativamente en el grupo sometido a la experiencia.

Tabla 3: Alumnos presentados a las pruebas de evaluación continua en el GE y en el GC

\begin{tabular}{|c|c|c|c|c|c|c|}
\hline \multirow{2}{*}{ Práctica } & \multicolumn{2}{|c|}{ Alumnos matriculados } & \multicolumn{2}{c|}{ Presentados } & \multicolumn{2}{c|}{ Porcentaje (\%) } \\
\cline { 2 - 7 } & GE & GC & GE & GC & GE & GC \\
\hline $2016 / 2017$ & 48 & 73 & 41 & 48 & $83.67 \%$ & $64.38 \%$ \\
\hline $2017 / 2018$ & 57 & 43 & 49 & 30 & $85.97 \%$ & $69.77 \%$ \\
\hline $2018 / 2019$ & 48 & 39 & 43 & 21 & $89.58 \%$ & $53.85 \%$ \\
\hline \multirow{2}{*}{ Teoría } & Alumnos matriculados & \multicolumn{2}{|c|}{ Presentados } & \multicolumn{2}{c|}{ Porcentaje (\%) } \\
\cline { 2 - 7 } & GE & GC & GE & GC & GE & GC \\
\hline $2016 / 2017$ & 48 & 73 & 41 & 48 & $83.67 \%$ & $65.75 \%$ \\
\hline $2017 / 2018$ & 57 & 43 & 50 & 30 & $85.96 \%$ & $69.77 \%$ \\
\hline $2018 / 2019$ & 48 & 39 & 30 & 11 & $62.50 \%$ & $28,21 \%$ \\
\hline
\end{tabular}

Por su parte, en la evaluación teórica, asumiendo la misma hipótesis, los valores obtenidos han sido: estadístico $Z$ = 2.18437 y p-valor 0.01447 para el curso 2016/2017; estadístico $Z=1.96877$ y p-valor 0.02449 
para el curso 2017/2018 y estadístico $Z=3.18692$ y p-valor 0.00072 en el curso 2018/2019. En este caso, con el mismo nivel de significación de $5 \%$, también se observa que hay diferencias significativas en todos los cursos analizados, lo que permite concluir de forma análoga al caso de la parte práctica de la materia, y que se encuentra en consonancia con los resultados obtenidos por otros autores (van Alten et al., 2019).

\section{Incidencia en el rendimiento académico de los estudiantes $\left(\mathrm{Pl}_{2}\right)$}

Para evaluar la incidencia de la nueva metodología frente a la metodología tradicional en el rendimiento académico de los alumnos se han utilizado los datos obtenidos de las pruebas de evaluación continua (práctica y teórica) realizadas a los dos grupos de alumnos en los tres cursos académicos. Con el fin de comprobar los supuestos de normalidad y homocedasticidad exigibles para la aplicación de una técnica paramétrica, se han sometido las calificaciones de los dos grupos en los tres cursos académicos a las pruebas de normalidad (Tabla 4). Aunque hay trabajos que afirman que la prueba de Shapiro-Wilk es la prueba de normalidad más poderosa, se ha constatado que los resultados son los mismos que con Kolmogorov-Smirnov con la corrección de Lillefors.

Tabla 4: Pruebas de normalidad. Resultados pruebas de evaluación práctica y teórica

\begin{tabular}{|c|c|c|c|c|c|c|}
\hline & \multicolumn{3}{|c|}{ Kolmogorov-Smirnov } & \multicolumn{3}{|c|}{ Shapiro-Wilk } \\
\hline & Estadístico & $\mathrm{gl}$ & Sig. & Estadístico & $\mathrm{gl}$ & Sig. \\
\hline \multicolumn{7}{|c|}{$\begin{array}{l}\text { Práctica } \\
\text { Curso 2016/2017 }\end{array}$} \\
\hline GC & .080 & 48 & .200 & .969 & 48 & .222 \\
\hline GE & .072 & 41 & .200 & .988 & 41 & .943 \\
\hline \multicolumn{7}{|c|}{ Curso 2017/2018 } \\
\hline GC & .139 & 30 & .143 & .953 & 30 & 201 \\
\hline GE & .125 & 49 & .055 & .963 & 49 & .124 \\
\hline \multicolumn{7}{|c|}{ Curso 2018/2019 } \\
\hline GC & .161 & 21 & .165 & .928 & 21 & .128 \\
\hline GE & .098 & 43 & $.200^{\mathrm{a}}$ & .950 & 43 & .057 \\
\hline \multicolumn{7}{|c|}{ Teoría } \\
\hline \multicolumn{7}{|c|}{ Curso 2016/2017 } \\
\hline GC & .070 & 48 & $200^{*}$ & .987 & 48 & .883 \\
\hline GE & .072 & 41 & $.200^{*}$ & .988 & 41 & .943 \\
\hline \multicolumn{7}{|c|}{ Curso 2017/2018 } \\
\hline$\overline{G C}$ & .118 & 30 & $.200^{*}$ & .945 & 30 & .121 \\
\hline GE & .142 & 50 & .013 & .923 & 50 & .003 \\
\hline \multicolumn{7}{|c|}{ Curso 2018/2019 } \\
\hline GC & .284 & 11 & .013 & .739 & 11 & .001 \\
\hline GE & .202 & 30 & .003 & .769 & 30 & .000 \\
\hline
\end{tabular}

Para los datos de las pruebas prácticas (Tabla 5), la comparación de medias se puede realizar mediante la prueba paramétrica $t$-Student al poder asumir normalidad en todos los casos, y que ha permitido plantear la siguiente hipótesis: $\mathrm{H}_{0}$ : Las medias de las calificaciones son iguales el GE y en el GC. Los resultados obtenidos (Tabla 5), para un nivel de significación de $5 \%$, permiten afirmar que en los dos primeros cursos (2016/2017 y 2017/2018) que no se observan diferencias significativas en el rendimiento académico de los grupos experimental y control, a pesar de que el promedio es superior los dos cursos en el grupo experimental. Sin embargo, para el curso 2018/2019 se observa que las puntuaciones del grupo experimental son significativamente superiores a las del grupo control.

En el caso de los datos de las pruebas de evaluación teórica, tanto atendiendo a Shapiro como a KolmogorovSmirnov (Tabla 5), en el primer curso podría aplicarse una técnica paramétrica, pero en los dos cursos siguientes no es posible al rechazar normalidad. Por este motivo, si bien en el caso del curso 2016/2017 se aplicó la prueba $t$ de Student, con el fin de homogenizar los resultados se ha optado por aplicar la técnica no paramétrica $U$ de Mann-Whitney para la comparación de medianas en todos los casos (Tabla 6). Los datos corroboran en todos los casos la misma conclusión (no hay diferencias significativas), si bien el promedio siempre es superior en el grupo sometido a la experiencia metodológica (FC). Resultados similares se encuentran en trabajos que abordan esta temática (Martínez-Jiménez y Ruiz-Jiménez, 2020). 
Tabla 5. Comparación de medias. Evaluación práctica

\begin{tabular}{|c|c|c|c|c|c|c|c|c|c|}
\hline & & \multicolumn{2}{|c|}{ Levene } & \multicolumn{6}{|c|}{ prueba t para la igualdad de medias } \\
\hline & & \multirow[t]{2}{*}{$F$} & \multirow[t]{2}{*}{ Sig. } & \multirow[t]{2}{*}{$t$} & \multirow[t]{2}{*}{ gl } & \multirow{2}{*}{$\begin{array}{c}\text { Sig. } \\
\text { (bilateral) }\end{array}$} & \multirow{2}{*}{$\begin{array}{c}\text { Diferencia de } \\
\text { medias }\end{array}$} & \multicolumn{2}{|c|}{$\begin{array}{l}95 \% \text { de intervalo de } \\
\text { confianza de la diferencia }\end{array}$} \\
\hline & & & & & & & & Inferior & Superior \\
\hline \multirow{2}{*}{$\begin{array}{c}\text { Curso } \\
2016 / 2017\end{array}$} & $\begin{array}{l}\text { Se asumen } \\
\text { varianzas iguales }\end{array}$ & .001 & .971 & -1.316 & 87 & .192 & -.81822 & -2.05423 & .41779 \\
\hline & $\begin{array}{l}\text { No se asumen } \\
\text { varianzas iguales }\end{array}$ & & & -1.320 & 85.694 & .190 & -.81822 & -2.05094 & .41450 \\
\hline \multirow{2}{*}{$\begin{array}{c}\text { Curso } \\
2017 / 2018\end{array}$} & \begin{tabular}{|l} 
Se asumen \\
varianzas iguales
\end{tabular} & 3.991 & .049 & -.695 & 77 & .489 & -.40429 & -1.56275 & .75418 \\
\hline & $\begin{array}{l}\text { No se asumen } \\
\text { varianzas iguales }\end{array}$ & & & -.655 & 50.393 & .516 & -.40429 & -1.64420 & .83563 \\
\hline \multirow{2}{*}{$\begin{array}{c}\text { Curso } \\
2018 / 2019\end{array}$} & $\begin{array}{l}\text { Se asumen } \\
\text { varianzas iguales }\end{array}$ & 9.657 & .003 & -3.906 & 62 & .000 & -2.46822 & -3.73141 & -1.20502 \\
\hline & $\begin{array}{l}\text { No se asumen } \\
\text { varianzas iguales }\end{array}$ & & & -3.367 & 28.225 & .002 & -2.46822 & -3.96916 & -.96728 \\
\hline
\end{tabular}

Tabla 6. Comparación de medianas. Evaluación teórica. a. Variable de agrupación: GC_GE

\begin{tabular}{|l|c|c|c|}
\hline & Curso 2016/2017 & Curso 2017/2018 & Curso 2018/2019 \\
\hline U de Mann-Whitney & 983.000 & 713.000 & 164.500 \\
\hline W de Wilcoxon & 1844.000 & 1178.000 & 629.500 \\
\hline Z & -.008 & -.368 & -.015 \\
\hline Sig. asintótica (bilateral) & .993 & .713 & .988 \\
\hline
\end{tabular}

Incidencia en la percepción de la metodología FC por parte de los estudiantes $\left(\mathrm{Pl}_{3}\right)$

En este caso, se trata de contrastar a través de las respuestas obtenidas en el cuestionario, antes y después de llevar a cabo la experiencia, si la opinión de los alumnos a propósito de FC se ve mejorada con la aplicación práctica de la clase invertida. Por tanto, solamente se lleva a cabo con el grupo experimental, con el grupo que ha sido sometido a la nueva metodología. Como se comentó con anterioridad, los ítems fueron agrupados (ver Tabla 2) con el objetivo de conocer la percepción de los alumnos en relación a dos cuestiones: aspectos actitudinales y motivacionales (frente a metodologías tradicionales) y desarrollo de competencias genéricas. Dado que se trata del mismo grupo evaluado en dos ocasiones diferentes, se plantea aplicar la prueba $t$ de muestras dependientes o apareadas (una prueba $t$ de mediciones repetitivas), sometiendo previamente los datos a las pruebas estadísticas para comprobar los supuestos de normalidad necesarios para su aplicación. Los resultados de las pruebas de normalidad de los datos obtenidos de las respuestas de los alumnos, atendiendo de forma separada a cada grupo de ítems (Tabla 7) muestran que en algún caso no es factible aplicar una técnica paramétrica. De ahí que, siendo conscientes de que en los supuestos de normalidad la aplicación de una técnica paramétrica o no paramétrica no va a afectar al resultado, se ha optado por homogeneizar la prueba para los dos grupos, utilizando en concreto la prueba de los rangos de Wilcoxon (Tabla 8).

Tabla 7. Pruebas de normalidad para cada grupo de ítems. *. Esto es un límite inferior de la significación verdadera.

\begin{tabular}{|c|c|c|c|c|c|c|c|}
\hline & \multirow[b]{2}{*}{ Grupo } & \multicolumn{3}{|c|}{ Kolmogorov-Smirnov } & \multicolumn{3}{|c|}{ Shapiro-Wilk } \\
\hline & & Estadístico & gl & Sig. & Estadístico & gl & Sig. \\
\hline \multirow{2}{*}{ Antes_Año1 } & 1 & .198 & 6 & $.200^{*}$ & .940 & 6 & .656 \\
\hline & 2 & .320 & 12 & .001 & .827 & 12 & .020 \\
\hline \multirow{2}{*}{ Despues_Año1 } & 1 & .170 & 6 & $.200^{*}$ & .951 & 6 & .749 \\
\hline & 2 & .165 & 12 & $.200^{*}$ & .963 & 12 & .823 \\
\hline \multirow{2}{*}{ Antes_Año2 } & 1 & .187 & 6 & $.200^{*}$ & .941 & 6 & .668 \\
\hline & 2 & .175 & 12 & $.200^{*}$ & .965 & 12 & .850 \\
\hline \multirow{2}{*}{ Después_año2 } & 1 & .274 & 6 & .179 & .785 & 6 & .043 \\
\hline & 2 & .157 & 12 & $.200^{*}$ & .950 & 12 & .642 \\
\hline \multirow{2}{*}{ Antes_Año3 } & 1 & .268 & 6 & $.200^{*}$ & .887 & 6 & .301 \\
\hline & 2 & .135 & 12 & $.200^{*}$ & .979 & 12 & .981 \\
\hline \multirow{2}{*}{ Después_Año3 } & 1 & .234 & 6 & $.200^{*}$ & .893 & 6 & .332 \\
\hline & 2 & .156 & 12 & $.200^{*}$ & .931 & 12 & .394 \\
\hline
\end{tabular}


Tabla 8. Prueba Wilcoxon para los dos grupos de ítems. a. Prueba de rangos con signo de Wilcoxon; b. Se basa en rangos negativos

\begin{tabular}{|c|l|c|c|c|}
\hline \multicolumn{2}{|l|}{ Grupo } & Después_Antes_Año1 & Después-Antes_Año2 & Después-Antes_Año3 \\
\hline \multirow{2}{*}{1} & Z & $-1.572^{\mathrm{b}}$ & $-2.201^{\mathrm{b}}$ & $-2.201^{\mathrm{b}}$ \\
\cline { 2 - 5 } & Sig. asintótica (bilateral) & .116 & .028 & .028 \\
\hline \multirow{2}{*}{2} & Z & $-2.746^{\mathrm{b}}$ & $-2.118^{\mathrm{b}}$ & $-3.059^{\mathrm{b}}$ \\
\cline { 2 - 5 } & Sig. asintótica (bilateral) & .006 & .034 & .002 \\
\hline
\end{tabular}

Los datos anteriores permiten tratar de dar respuesta a las dos preguntas de investigación propuestas $\left(\mathrm{PI}_{3-1}\right.$ y $\mathrm{Pl}_{3-2}$ ), planteando las hipótesis respectivas: 1) $\mathrm{H}_{0}$ : Las medias de las respuestas en relación a "aspectos actitudinales y motivacionales" es la misma antes y después de la experiencia; 2) $\mathrm{H}_{0}$ : Las medias de las respuestas en relación a las "competencias adquiridas" es la misma antes y después de la experiencia

En base a los resultados obtenidos en el Grupo 1 "Aspectos actitudinales y motivacionales", en el primer curso se ha de aceptar que, si bien ha habido una mejora en las expectativas de los alumnos, ésta no ha sido significativa. Sin embargo, en los dos cursos siguientes se puede afirmar que ha habido un incremento significativo en las expectativas de los alumnos en relación a cuestiones actitudinales y motivacionales a través de la aplicación de la metodología FC. Los datos para los ítems del Grupo 2 "Competencias adquiridas" permiten afirmar que los alumnos manifiestan un incremento en las competencias adquiridas con la metodología evaluada en todos los cursos analizados. Estos resultados concuerdan con varios trabajos al respecto, como los recogidos en el estudio de Van Alten et al. (2019). En relación a las competencias, Estriegana et al. (2019) concluyen además -como es nuestro caso- que no coincide exactamente con los resultados académicos medidos durante el proceso de evaluación, lo que redunda en la necesidad de un análisis separado. En relación al cuestionario final, como se comentó anteriormente, se reflejó un nuevo ítem o pregunta con la siguiente redacción: "Me gustaría mantener esta metodología frente a la metodología tradicional en el resto del curso". Con este proceder se trata de conocer no solamente la percepción del alumnado hacia la metodología FC una vez llevada a cabo la experiencia, sino también su predisposición final hacia la misma. Los datos resumidos de las respuestas obtenidas se muestran en la Tabla 9.

Tabla 9. Porcentajes relativos de las frecuencias, respuesta ítem 19

\begin{tabular}{|l|c|c|c|}
\hline Respuesta & Año 1 & Año 2 & Año 3 \\
\hline Muy poco & $0.00 \%$ & $0.00 \%$ & $0.00 \%$ \\
\hline Poco & $0.00 \%$ & $0.00 \%$ & $0.00 \%$ \\
\hline Suficiente & $3.45 \%$ & $3.85 \%$ & $3.85 \%$ \\
\hline Bastante & $17.24 \%$ & $23.08 \%$ & $15.38 \%$ \\
\hline Mucho & $79.31 \%$ & $73.08 \%$ & $80.77 \%$ \\
\hline
\end{tabular}

Un simple análisis de frecuencias da como resultado que mayoritariamente están predispuestos con un grado de "Bastante" o "Mucho" a que toda la asignatura se imparta bajo la metodología propuesta. De hecho, los porcentajes acumulados para estas dos valoraciones ascienden al 96,55\% en el curso 2016/2017, a 96,16\% en el $2017 / 2018$ y a $96,15 \%$ en el curso $2018 / 2019$. En otro orden de cosas, no ha habido respuestas en ninguno de los cursos, que opinen que no consideren esta metodología como válida ("Poco" o "Muy poco").

En un análisis crítico del trabajo podemos señalar como limitación principal que el objeto del mismo se circunscribe a un caso particular en los estudios de Grado de la enseñanza universitaria en el caso español, lo cual por otra parte le otorga una cierta contribución particular en el mismo sentido. Adicionalmente, no se trata de un cuasi-experimento en condiciones definidas por los autores, sino que atiende a una realidad mantenida en tres cursos académicos. Asimismo, estas limitaciones abren una ventana de oportunidad de exploración de desarrollo futuro al objeto de su generalización en otros ámbitos de estudio.

\section{CONCLUSIONES}

De acuerdo al trabajo presentado y a los resultados obtenidos, se pueden plantear las siguientes conclusiones principales:

1.-La utilización de la metodología de aula invertida frente a la metodología tradicional ha supuesto mayor motivación de los estudiantes hacia la asignatura, medida en términos de su participación en el proceso de evaluación continua, en los tres cursos académicos analizados, tanto en la parte teórica como práctica de la asignatura. 
2.-El rendimiento académico de los estudiantes que han sido sometidos a la metodología de aula invertida, medido en términos de sus calificaciones en la prueba de evaluación continua, ha sido superior en los tres cursos analizados a las obtenidas por el grupo control, tanto en la prueba práctica como teórica. No obstante, desde el punto de vista estadístico dicha mejoría no es significativa.

3.-La valoración previa de los estudiantes sobre la metodología de aula invertida ha mejorado con la experiencia en términos generales, tanto en relación a "Cuestiones actitudinales y motivacionales" como a las "Competencias adquiridas", lo que permite concluir que el aula invertida puede resultar un incentivo a la actitud y motivación de los estudiantes, así como un elemento facilitador de la adquisición de competencias desde la perspectiva del alumnado.

\section{REFERENCIAS}

Abeysekera, L., y Dawson, P., Motivation and cognitive load in the flipped classroom: definition, rationale and a call for research, https://doi.org/10.1080/07294360.2014.934336, High. Educ. Res. Dev., 34(1), 1-14 (2015)

Bergmann, J., y Sams, A., Flip your classroom: reach every student in every class every day, $1^{\underline{a}}$ ed., International Society for Technology in Education (2012)

Brown, C. A., Danvers, K., y Doran, D.T., Student perceptions on using guided reading questions to motivate student reading in the flipped classroom, https://doi.org/10.1080/09639284.2016.1165124, Account. Educ., 25(3), 256-271 (2016)

Cheng, L., Ritzhaupt, A.D., y Antonenko, P., Effects of the flipped classroom instructional strategy on students' learning outcomes: a meta-analysis, https://doi.org/10.1007/s11423-018-9633-7, Educ. Technol. Res. Dev., 67(4), 793-824 (2019)

Estriegana, R., Medina-Merodio, J.-A., y Barchino, R., Analysis of competence acquisition in a flipped classroom approach, https://doi.org/10.1002/cae.22056, Comput. Appl. Eng. Educ., 27(1), 49-64 (2019)

Flores, Ò., Del-Arco, l., y Silva, P., The flipped classroom model at the university: analysis based on professors' and students' assessment in the educational field, https://doi.org/10.1186/s41239-016-0022-1, Int. J. Educ. Technol. High. Educ., 13(1), 21 (2016)

Galindo-Domínguez, H., y Bezanilla, M.J., A systematic review of flipped classroom methodology at university level in Spain, https://doi.org/10.24310/innoeduca.2019.v5i1.4470, Innoeduca: International Journal of Technology and Educational Innovation (IJERI), 5(1), 81-90 (2019)

Gutiérrez-Monsalve, J.A., Garzón, J., y Segura-Cardona, A.M., Factores asociados al rendimiento académico en estudiantes universitarios. https://doi.org/10.4067/S0718-50062021000100013, Form. Univ., 14(1), 13-24 (2021)

Jamaludin, R., Md Osman, S.Z., Wan Yusoff, W.M., y Azwa Jasni, N.F., Flipped: a case study in fundamental of accounting in Malaysian Polytechnic, https://doi.org/10.20448/journal.509/2016.3.1/509.1.23.31, J. Educ. Elearn. Res., 3(1), 23-31 (2016)

Jensen, J.L., Holt, E.A. y otros tres autores, Investigating strategies for pre-class content learning in a flipped classroom, https://doi.org/10.1007/s10956-018-9740-6, J. Sci. Educ. Technol., 27(6), 523-535 (2018)

Karanicolas, S., Snelling, C., y Kemp, E., Flipped classroom design framework aligned with Bloom's modified taxonomy, https://www.adelaide.edu.au (2018)

Kim, M.K., Kim, S.M., Khera, O., y Getman, J., The experience of three flipped classrooms in an urban university: an exploration of design principles, https://doi.org/10.1016/j.iheduc.2014.04.003, Internet High Educ., 22, 37-50 (2014)

Lento, C., Promoting active learning in introductory financial accounting through the flipped classroom design, https://doi.org/10.1108/JARHE-01-2015-0005, J. Appl. Res. High. Educ., 8(1), $72-87$ (2016)

Limniou, M., Schermbrucker, I., y Lyons, M., Traditional and flipped classroom approaches delivered by two different teachers: the student perspective, https://doi.org/10.1007/s10639-017-9636-8, Educ. Inf. Technol., 23(2), 797-817 (2018)

Little, C., The flipped classroom in further education: literature review and case study, https://doi.org/10.1080/13596748.2015.1063260, Research in Post-Compulsory Education, 20(3), 265-279 (2015)

Lundin, M., Bergviken Rensfeldt, A., y otros tres autores, Higher education dominance and siloed knowledge: a systematic review of flipped classroom research, https://doi.org/10.1186/s41239-018-0101-6, Int. J. Educ. Technol. High. Educ., 15(1), 20 (2018)

Martínez-Jiménez, R., y Ruiz-Jiménez, M.C., Improving students' satisfaction and learning performance using flipped classroom, https://doi.org/10.1016/j.jime.2020.100422, Int. J. Manag. Educ., 18(3), 100422 (2020)

McNally, B., Chipperfield, J., y otros diez autores, Flipped classroom experiences: student preferences and flip strategy in a higher education context, https://doi.org/10.1007/s10734-016-0014-z, High. Educ., 73(2), 281-298 (2017)

Mosteller, F., "The muddiest point in the lecture" as a feedback device, The Journal of the Harvard-Danforth Center, 3 , 10-21 (1989) 
Nagy, J.T., Evaluation of online video usage and learning satisfaction: an extension of the technology acceptance model, https://doi.org/10.19173/irrodl.v19i1.2886, The International Review of Research in Open and Distributed Learning (IRRODL), 19(1), 160-184 (2018)

Oblinger, D.G., y Oblinger, J. L. (eds.), Educating the Net generation, $1^{\mathrm{a}}$ ed., Educause (2005)

Poy Castro, R., Mendaña Cuervo, C., y otros 3 autores, Un instrumento para evaluar las expectativas de alumnos ante la implementación de una metodología activa. III TeLe(In)2 Conference Proceedings, https://issuu.com/rabanaln/docs/actas_20del_20iii_20congreso_20tele (2017)

Prieto, A., Barbarroja Escudero, J., Corell, A., y Álvarez Álvarez, S., Eficacia del modelo de aula invertida (flipped classroom) en la enseñanza universitaria: una síntesis de las mejores evidencias, https://doi.org/10.4438/1988-592XRE-2021-391-476. Rev. Educ., 391, 49-177 (2021)

Rivero-Guerra, A.O., Práctica de laboratorio de granos de almidón en un curso de universitario de botánica general: una experiencia de clase invertida. https://doi.org/10.4067/s0718-50062018000100087, Form. Univ., 11(1), 87-104 (2018)

Roach, T., Student perceptions toward flipped learning: new methods to increase interaction and active learning in Economics, https://doi.org/10.1016/j.iree.2014.08.003, Int. Rev. Econ. Educ., 17, 74-84 (2014)

Ros Clemente, M.I., Conesa Pérez, M.C., Martínez Franco, C.M., y Meseguer Martínez, Á., Aplicando la clase invertida: contabilidad, in Ros García (ed.), Aplicando la clase invertida en empresa, economía y turismo, 53-66, Dykinson, Madrid, España (2018)

Sohrabi, B., y Iraj, H., Implementing flipped classroom using digital media: a comparison of two demographically different groups perceptions, https://doi.org/10.1016/j.chb.2016.02.056, Comput. Hum. Behav., 60, 514-524 (2016)

Steed, A., The flipped classroom, Teaching Bus. Econ., 16(3), 9-11 (2012)

Tse, W.S., Choi, L.Y.A., y Tang, W.S., Effects of video-based flipped class instruction on subject reading motivation, https://doi.org/10.1111/bjet.12569, Br. J. Educ. Technol., 50(1), 385-398 (2019)

Uzunboylu, H., y Karagozlu, D., Flipped classroom: a review of recent literature, https://doi.org/10.18844/wjet.v7i2.46, World Journal on Educational Technology (WJET), 7(2), 142-147 (2015)

van Alten, D.C.D., Phielix, C., Janssen, J., y Kester, L., Effects of flipping the classroom on learning outcomes and satisfaction: a meta-analysis, https://doi.org/10.1016/j.edurev.2019.05.003, Educ. Res. Rev., 28 (2019)

Zainuddin, Z., y Halili, S.H., Flipped classroom research and trends from different fields of study, https://doi.org/10.19173/irrodl.v17i3.2274, International Review of Research in Open and Distributed Learning (IRRODL), 17(3), 313-340 (2016) 